\title{
Scaling of Discomfort Sensations in the Conditions of Performing Office Visual Tasks
}

\author{
Georgy Boos ${ }^{1}$, Vladimir Budak ${ }^{1}$, Ekaterina Ilyina ${ }^{1}$ and Tatyana Meshkova ${ }^{1}$ \\ ${ }^{1}$ National Research University “Moscow Power Engineering Institute”, Krasnokazarmennaya 14, Moscow, \\ 111250, Russia
}

\begin{abstract}
Currently, computer graphics programs (CG) as a tool for designing lighting installations allows us to move to a fundamentally new approach to assessing the quality of lighting: to move away from designing based on illuminance values to designing based on synthetic images or lighting design. Modern CG programs allow us to calculate the spatial-angular distribution of luminance (SADL). Still, some criterion is needed for the assessment, which will allow us to uniquely determine the quality of lighting in terms of luminance distribution.

In this paper, we consider constructing a physiological model of the visual sense scale for assessing the quality of lighting according to SADL based on an experiment to assess visual sense from the luminance of a light source in the field of view on a uniform background with different luminance. As a result of the work done, it is proposed to use a scale of sensations in the form of a certain space. The axis of space is the threshold contrast for the resulting sense of discomfort, depending on the observation conditions. All other sensations are separated from the axis at some distance. Threshold contrasts, at which visual sensations arise within the framework of the proposed scale, can be used in the future to assess the storm in the lighting scene using a new criterion and will allow analysing the quality of lighting using CG methods.
\end{abstract}

\section{Keywords}

Visual sensation scale, discomfort, discomfort score, UGR

\section{Introduction}

Currently, computer graphics programs (CG) have become the main tool for designing lighting installations (LI). The use of CG determined a fundamentally new approach to design: the synthetic image of the projected installation allowed us to move away from designing for illuminance values to designing images - lighting design. In the modern understanding of lighting design, the design is conducted on the spatial-angular distribution of luminance (SADL) [1], which allows us to calculate modern CG programs. At the same time, there is an important question: how to evaluate the quality of the obtained luminance distribution in a synthetic lighting scene? The normalization of lighting quality should not be based on distributions but on the value of some criterion that will allow you to uniquely find the quality of lighting in terms of luminance distribution. Now, there is no means of assessing the quality according to the calculated SADL. As a result, recommendations have not been developed, and there is no rationing according to the criterion showing which luminance distribution can be considered pleasant and which is uncomfortable.

Human-centric lighting (non-human-oriented lighting) is a modern motto that is not yet feasible in practice. We know little about the non-visual effect of light on a person. While we have studied the visual effect well, we do not know how to express it unambiguously through the photometric

GraphiCon 2021: 31st International Conference on Computer Graphics and Vision, September 27-30, 2021, Nizhny Novgorod, Russia EMAIL: BoosGeorV@mpei.ru(G.Boos); budakvp@gmail.com (V.Budak); kitesika@gmail.com (E.Ilyina); tvmesh@mail.ru (T.Meshkova) ORCID: 0000-0001-9725-4266 (G.Boos); 0000-0003-4750-0160 (V.Budak); 0000-0003-0783-0931 (E.Ilyina); 0000-0003-1008-2161 (T.Meshkova)

(c) (i) 2021 Copyright for this paper by its authors.

Use permitted under Creative Commons License Attribution 4.0 International (CC BY 4.0).

CEUR Workshop Proceedings (CEUR-WS.org) 
characteristics of the light field [2]. Generally HCL is considered from the point of influence of the spectral density of light and of vertical illuminance in the eye. In [3], the luminance distribution is considered as weighty parameter for human's health.

The modern understanding of high-quality lighting is based on normalized parameters: quantitative and qualitative. The quality of lighting (in terms of light distribution) is related to the concept of visual comfort. The simplest definition of visual comfort sounds like the absence of visual discomfort. On the one hand, some lighting conditions can cause discomfort. On the other hand, there is a positive sense of comfort that occurs after eliminating all sources of discomfort. Currently, the assessment of indoor lighting is carried out based on illuminance values. At the same time, the eye reacts to luminance. A highly uneven (arbitrary) distribution of luminance in the scene and the presence of a wide range of luminance in the field of view can be a source of discomfort [2], whereas with a standard assessment through the illuminance of the same scene, the norms can be met. Thus, the issue of the occurrence of discomfort from lighting has not been fully studied. Still, it can be assumed that there is a certain connection between the resulting sense of comfort/discomfort and the distribution of luminance in space.

Currently, there is no exact expression for evaluating the quality of lighting with an arbitrary luminance distribution, including glare light sources and glare. In [4], it is proposed to use the methods of CG modelling to find the quality of indoor lighting according to the SADL. The methods applicable to diffuse surfaces do not consider the factors associated with a sharp difference in luminance of the observed light source or glare on the background of the working plane. So, the question of choosing a comfortable difference (contrast) today is very relevant in such tasks. Scenes with diffuse surfaces can include sharp luminance differences (up to 20 times). It can be seen in places where two surfaces with different reflection coefficients come into contact. In most cases these differences are not equal to ones occurred when a source enters the field of view because the luminance levels of the illuminated surfaces are obviously lower than the luminance of the light sources. The task of finding the visual perception of lighting belongs to the category of psychophysical tasks. Such problems do not have an exact solution, so it is possible to build a model that will predict the sensation with some accuracy and thereby characterize the quality of the lighting scene. In addition, within the framework of this work, the issue of comfort/discomfort of lighting with light source observed in the field of view is studied. This luminance difference is important for evaluating the quality of indoor lighting according to the SADL.

Studies [5,6] have shown that too high unevenness can be excessively stimulating, and irritating, and uniform illumination creates a boring visual environment. It is noted in [2] that in the working area, the uniformity of illuminance, measured as the ratio of the minimum/average, is not complete. It is important to consider where the minimum and maximum illuminance occur, and the rate of luminance change between them. Therefore, the concept of a gradient (difference) of luminance defined through the threshold luminance contrast on the discomfort scale is an urgent task. CG makes it easy to estimate the luminance gradient from a calculated image or a real photo, but the question is how to rank the sensations. Simply put, what contrast (difference) of luminance in the scene will cause this or that sensation?

The modern approach to assessing the discomfort of lighting is based on the calculation of the unified glare rating (UGR), which allows you to make a forecast of the blinding effect of lighting installations on the psychophysical scale of the discomfort scale. In fact, UGR is a measure of the sense of this scale. The UGR values are linked to the actual values of the luminance of the light source $\left(L_{S}\right)$ and the luminance of the adaptation $\left(L_{a d}\right)$, as well as to the size and position of the source compared to the observation line. When calculating UGR, the average background luminance is taken as the adaptation luminance, without considering the luminance of the light sources. Values below 10 indicate that the lighting conditions will be perceived as comfortable, and above 28 there will be a blinding effect for most observers. At the same time, UGR does not allow considering reflected glare, and has a limit on the size of light sources. According to [7] the calculation of UGR based on luminance maps is in good agreement with the calculation based on intensity distribution of light. But for non-uniform luminaires, UGR values obtained from luminance maps result in a wide range of values depending on the grouping algorithm. So, the actual UGR formula is not robust for using calculations based on luminance maps. This means that popular non-uniform LED's luminaries can't be estimated correctly by this way. 
It should be noted that the visual perception of lighting depends on the adaptation of the visual system, on the environment and is determined by our current knowledge and our experience. The study of visual perception is based on simple tasks reduced to the maximum number of controlled parameters. The classical one in this case is an experimental setup with a glare light source on a uniform background, creating a luminance difference in the observer's field of view [8]. The first psychophysical scale of Lekiesh and Holladay was built on such an installation [9], who tried to find the relationship between the numerical values of $L_{S}$ and $L_{a d}$, and a set of human reactions, expressed in the categories of comfort of lighting. This scale consisted of 14 nominal categories: "barely noticeable", "most pleasant", "still pleasant", "on the verge of pleasant", "on the verge of comfortable", "very comfortable", "still comfortable", "less comfortable", "uncomfortable", "noticeably unpleasant", "unpleasant", "completely uncomfortable", "unpleasant/unbearable" and "painful".

In connection with recent studies [10], discomfort may arise not only due to the presence of glare light sources, but also since the environment around is boring, monotonous. That is why it is important to define a scale much wider than the UGR discomfort scale, that is, to evaluate other sensations, for example: the threshold for detecting light source, comfort, discomfort, distraction, and soreness. To assess the quality of luminance according to the SADL, it is necessary to create such a scale. The occurrence of any sensation, not only discomfort - is the triggering of a certain threshold, after which the observer feels a change in his attitude to the distribution of luminance in space. Our hypothesis is that when a certain threshold contrast is reached, the observer has a sensation defined on the scale of visual perception of the luminance distribution. Such a scale will display the relationship between the numerical values of the luminance of the light source and the luminance of the adaptation in the resulting image and the set of human reactions. Since the scale of sensations refers to the scale of verbal categories, the observer experiences difficulties in interpreting his sensations if there are many of them, as was the case in the above-mentioned Lekiesh Holladay's scale. It is for these reasons that within the framework of our study [11], only five sensations were identified: "barely noticeable" - the glare light source becomes noticeable at the time of reading the inscription, in fact, this is the threshold for detecting a signal, "comfortable" - it is pleasant to read the inscription and the light source does not distract, "uncomfortable" - the sense of a "buzzing fly", does not interfere with reading, but already attracts attention, "unpleasant" - distracts from reading the inscription, "unbearable" means painful. Such a scale should not be limited to finding the boundary of discomfort or the threshold contrast that causes discomfort. It should consider states from the appearance of a sense of the presence of a light source (when the event becomes noticeable) to its limit (when it becomes unbearable). Note that we are talking about an ordinal scale, since all the sensations listed above arise in order. In addition, we note that it is limited by a certain interval, that is, there is a restriction from below - the threshold for detecting the signal and a restriction from above - this is the luminance distribution, at which performing visual work becomes impossible.

Since the sensitivity of the eye varies depending on the luminance level, for each interval of the scale, the variance also increases with the increase in the average luminance value. In [12], the scale of sensations was compared with the values of the discomfort index M (like the UGR discomfort scale), calculated using an empirical formula for a single glare source according to the system of categorical judgments. Since the discomfort indicator had a normal distribution of ratings on the scale, it was proposed to find the numerical boundaries of the intervals of subjective categories in units of standard deviation and find the numerical boundaries of the intervals for a certain probability value, for example, $p=0.5$ or 0.7 . This approach will be applied later in the construction of our scale.

The purpose of this work is to build a physiological model of the scale for evaluating lighting scenes according to SADL based on empirical data obtained from an experiment to evaluate the reaction of observers depending on the luminance of a glare light source. The paper solves the problem of choosing a metric and finding the numerical boundaries of the intervals of subjective categories in units of standard deviation for a certain probability value.

\section{Methods}


An experimental installation like the installation of Lekiesh and Guth but with a large range of luminance and on modern LED light sources [13] was installed at the Department of Lighting Engineering of the National Research University "Moscow Power Engineering Institute". 9 students (7 guys and 2 girls) participated in the experiment. 367 answers for different conditions were obtained. The observer was found at $1.0 \mathrm{~m}$ from the installation and considered a colour palette of 24 colours measuring $12 \times 8 \mathrm{~cm}^{2}$, the centre of which was at the level of the line of sight. At the same time, the glare light source was found $10^{\circ}$ above the line of view on the background of an illuminated white surface measuring $2 \times 2 \mathrm{~m}^{2}$. The experiment was conducted in a classroom in the daytime with the windows curtained with blinds.

According to the definition of the Commission Internationale de l'eclairage (CIE), background luminance is formed by the luminance of all objects in the observer's field of view, except for glare sources and glare. In our case, the average background luminance was found using the Aftab Alpha program from three images of the scene captured in the format. NEF with different exposure settings $+1 \mathrm{E}, 0$ and $-1 \mathrm{E}$ (see Fig. 1). These images were converted to the HDR format (images with an extended luminance range). To normalize the images by luminance, the measured luminance values at several points of the scene were used using the Konika Minolta LS-100 luminance meter. The uniformity of the background luminance $L_{b}=L_{\max } / L_{a v}$ of this surface is $75 \%$. In the installation, the light source varied in the range from 67 to $56000 \mathrm{~cd} / \mathrm{m}^{2}$ and $L_{a d}$-from 21 to $190 \mathrm{~cd} / \mathrm{m}^{2}$.

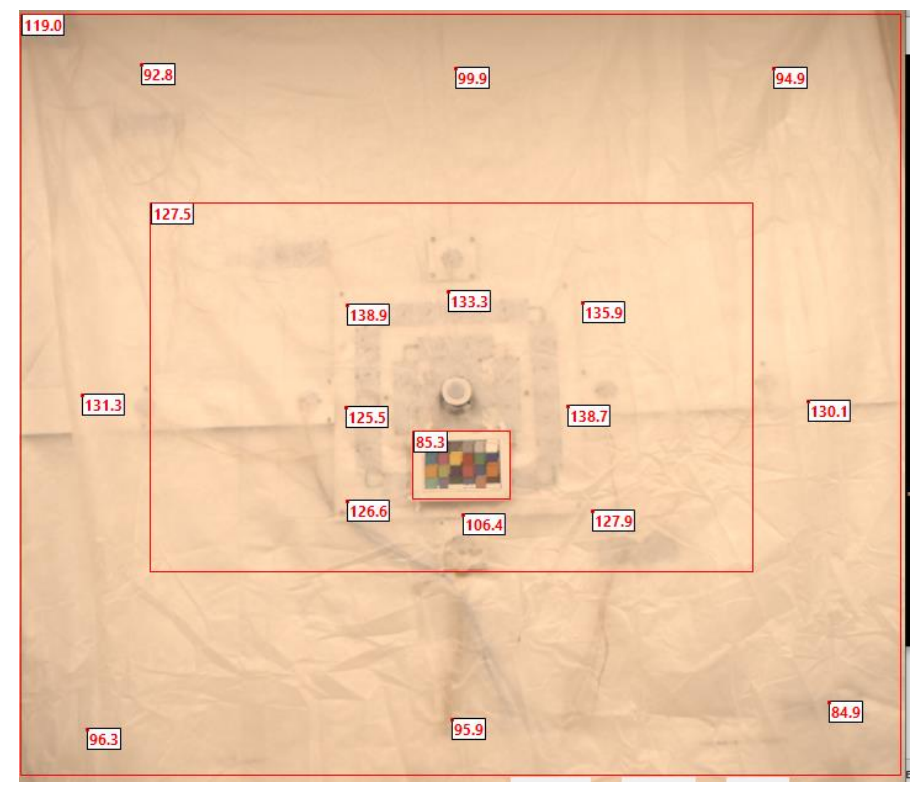

Figure 1: Photo of the installation for determining contrasts and calculating the average luminance of the adaptation, object and background in the program Aftab Alpha

The observer smoothly changed the luminance of the light source at a constant background luminance and recorded the $L_{S}$ readings. Between measurements with different $L_{a d}$ levels, a pause of 5 minutes was made so that the observer's eyes had time to rest.

In our installation, when the luminance of the source increased, the luminance of the field around it increased, which affected the background luminance, and, so, the adaptation luminance. At the same time, the background luminance at the background luminance $L_{b}=128 \mathrm{~cd} / \mathrm{m}^{2}$ increased by no more than $5 \mathrm{~cd} / \mathrm{m}^{2}$, which is $3.9 \%$. Therefore, this slight increase in the background luminance was not considered. The light source luminance may also contribute to the adaptation luminance. Since the luminous adaptation with increasing luminance occurs within a few minutes, and the speed of measuring threshold sensations was from 15 to $30 \mathrm{sec}$., the eye did not have time to adapt to the changing luminance of the light source. The light source luminance was also not considered in the calculation of the adaptation luminance since it was impossible to estimate it.

To study the discomfort from a lighting installation in a lighting scene, the following five categories were used as a psychometric scale of visual perception: "barely noticeable", "comfortable", "uncomfortable", "unpleasant" and "unbearable". So, the "barely noticeable" criterion is the threshold 
for detecting a signal. This category served as a reference point for constructing the scale. The category "unbearable" is a pain threshold or a threshold at which further performance of a visual task is impossible, or rather, it is the boundary value of the scale, respectively. The most difficult category is "comfortable", since it is difficult to describe it and, therefore, each observer formed his own understanding of comfort. Nevertheless, all observers were guided by the description of a comfortable sunbeam. A clearer category is "uncomfortable". It was associated with the description of a "buzzing fly". The "unpleasant" category meant that the IP was distracting from seeing the palette.

The calculation of the contrast at which a particular sensation occurred was performed according to the formula:

$$
C=\frac{\Delta L}{L_{a d}}=\frac{L_{S}-L_{a d}}{L_{a d}}=C_{1}-1,
$$

where the ratio of the luminance of the light source to the luminance of the background can be expressed as follows:

$$
C_{1}=\frac{L_{S}}{L_{a d}} .
$$

In this case, the contrast $C_{1}$ does not find the visibility of the object against a certain background, but the sensation that the light source causes while in the field of view. In our case, each sensation can be described by its value $\mathrm{C}_{1}(i)$, where $i$ is the ordinal number of the category.

\section{Results}

The results were obtained in an experiment with a glare source found $10^{\circ}$ above the line of sight. The average threshold values of $C_{1}$ for each sensation and standard deviations were obtained under the condition of a uniform background, on which there is one glare source, and for a simple visual task seeing the palette - are shown in Table 1. Table 1 shows that the largest standard deviations are seen in the category of the "comfortable" scale. This is since a comfortable luminance contrast is a very subjective concept. Whereas "uncomfortable" contrasts have a smaller spread.

We will construct graphs of the inverse normal distribution of the probability of sensations for the luminance of the adaptation $L_{a d}=21 \mathrm{~cd} / \mathrm{m}^{2}$ (see Fig. 2). The graphs are linear in nature, which speaks in favour of the normal distribution.

Table 1

Average threshold contrasts for each sensation for different luminance levels of adaptation

\begin{tabular}{cccccc}
\hline $\begin{array}{c}\text { Lad, } \\
\text { cd/m2 }\end{array}$ & $\begin{array}{c}\text { barely } \\
\text { noticeable }\end{array}$ & comfortable & uncomfortable & unpleasant & unbearable \\
\hline 21 & $1.05 \pm 0.2$ & $7.03 \pm 5.28$ & $59.77 \pm 44.99$ & $263.17 \pm 190.77$ & $734.73 \pm 398.60$ \\
87.0 & $0.88 \pm 0.17$ & $9.75 \pm 8.14$ & $63.99 \pm 47.64$ & $160.44 \pm 81.27$ & $296.47 \pm 79.57$ \\
128.0 & $0.98 \pm 0.22$ & $15.28 \pm 17.05$ & $62.35 \pm 35.87$ & $138.41 \pm 86.31$ & $305.43 \pm 119.69$ \\
152.0 & $0.91 \pm 0.21$ & $12.15 \pm 15.11$ & $58.56 \pm 23.17$ & $118.71 \pm 37.69$ & $266.09 \pm 59.80$ \\
180.0 & $0.99 \pm 0.19$ & $12.34 \pm 7.16$ & $52.74 \pm 26.93$ & $118.17 \pm 46.36$ & $208.55 \pm 51.97$ \\
190.0 & $1.06 \pm 0.27$ & $12.85 \pm 9.11$ & $55.53 \pm 23.50$ & $113.04 \pm 21.42$ & $196.78 \pm 17.64$ \\
\hline
\end{tabular}

The linearity is preserved, as can be seen from Fig. 2 for different luminance levels. Only the slope of the curve changes. With an increase in the adaptation luminance, the angle of inclination of the line decreases. Thus, we will take the category of uncomfortable contrast as the centre of the scale. We will analyse the distribution of other curves of sensations compared to discomfort. To do this, we divide the value of the threshold contrast of each sensation by the value of the uncomfortable contrast for each observer separately. And we will plot the curves of sensations with the centre at the point of discomfort (see Fig. 3). Note that by increasing the luminance of the adaptation, their angle of inclination changes 
when passing through discomfort. That is, the sense between the sense is unbearable and uncomfortable, the distance in the space of the scale decreases with increasing the adaptation luminance.
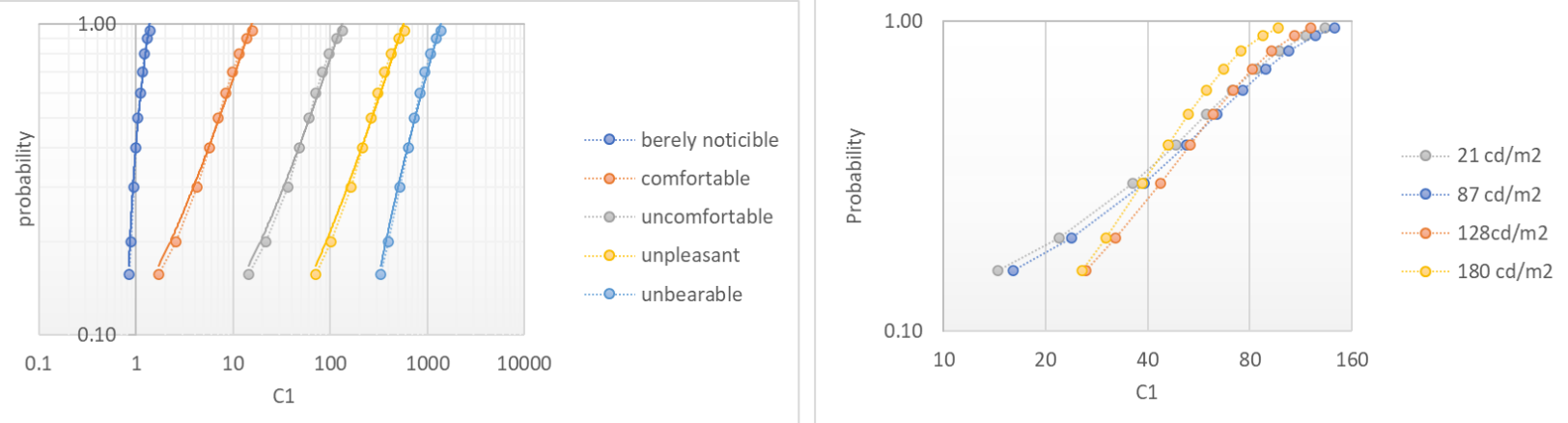

Figure 2: On the left, graphs of the inverse normal distribution of threshold contrasts of sensations at luminance $L_{a d}=21 \mathrm{~cd} / \mathrm{m}^{2}$. On the right, only for "uncomfortable" contrast at $L_{a d}=21,87,128,180$ $\mathrm{cd} / \mathrm{m}^{2}$, respectively

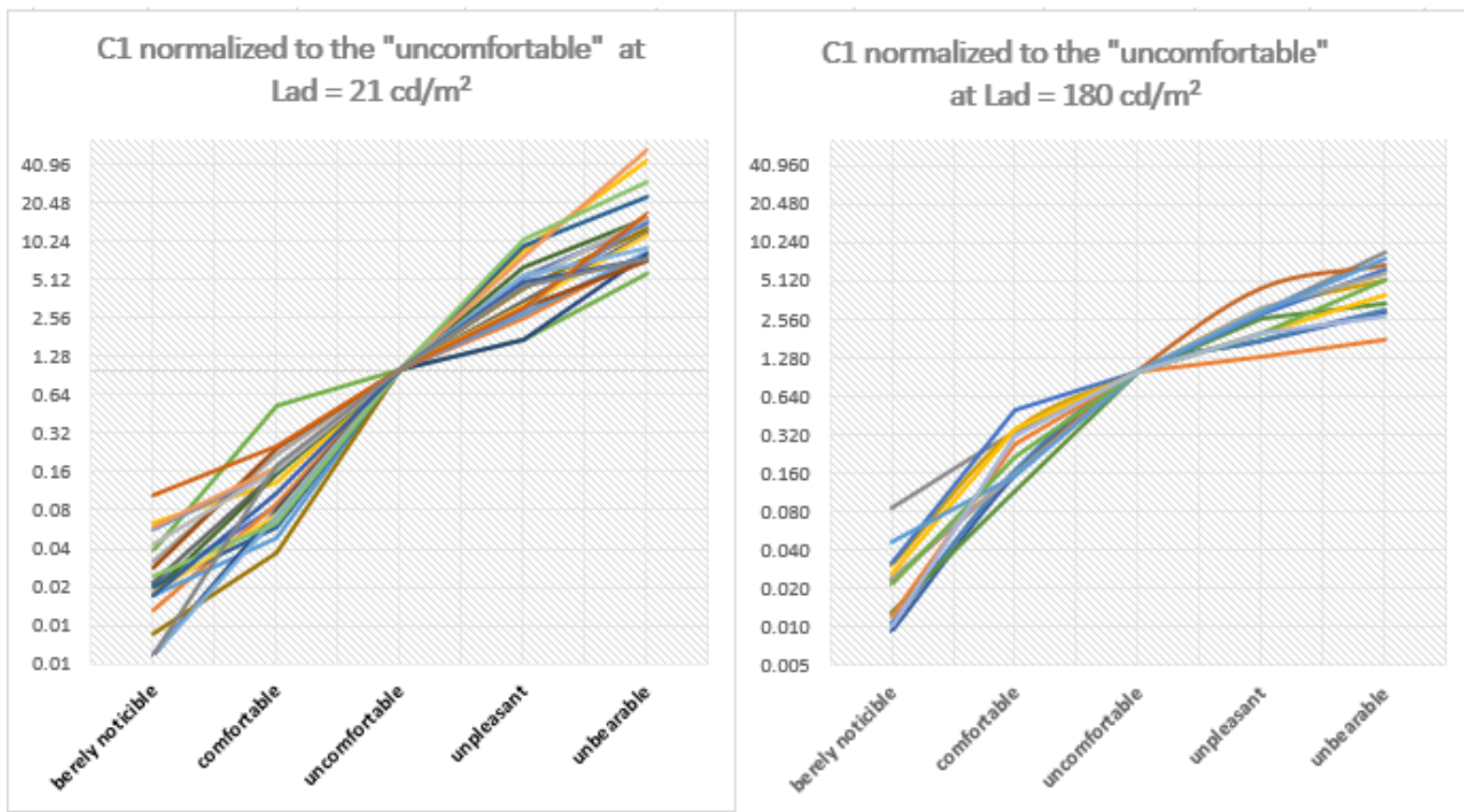

Figure 3: The scale of sensations according to the contrast of the luminance difference $C_{1}$, normalized to the "uncomfortable» $\mathrm{C} 1$ at luminance adaptations of 21 and $180 \mathrm{~cd} / \mathrm{m}^{2}$ respectively (logarithmic scale is used)

That is, the scale of sensations will be tied to the adaptation luminance and cannot be expressed by a single number for the entire space. Figure 4 shows how the distance between the axis of uncomfortable contrast, taken as the centre of reference, and other sensations changes depending on the adaptation luminance. The curves above the axis of discomfort can be given by equations with a high degree of correlation. The curves under the axis responsible for the discomfort can be approximated with a lower degree of correlation. 


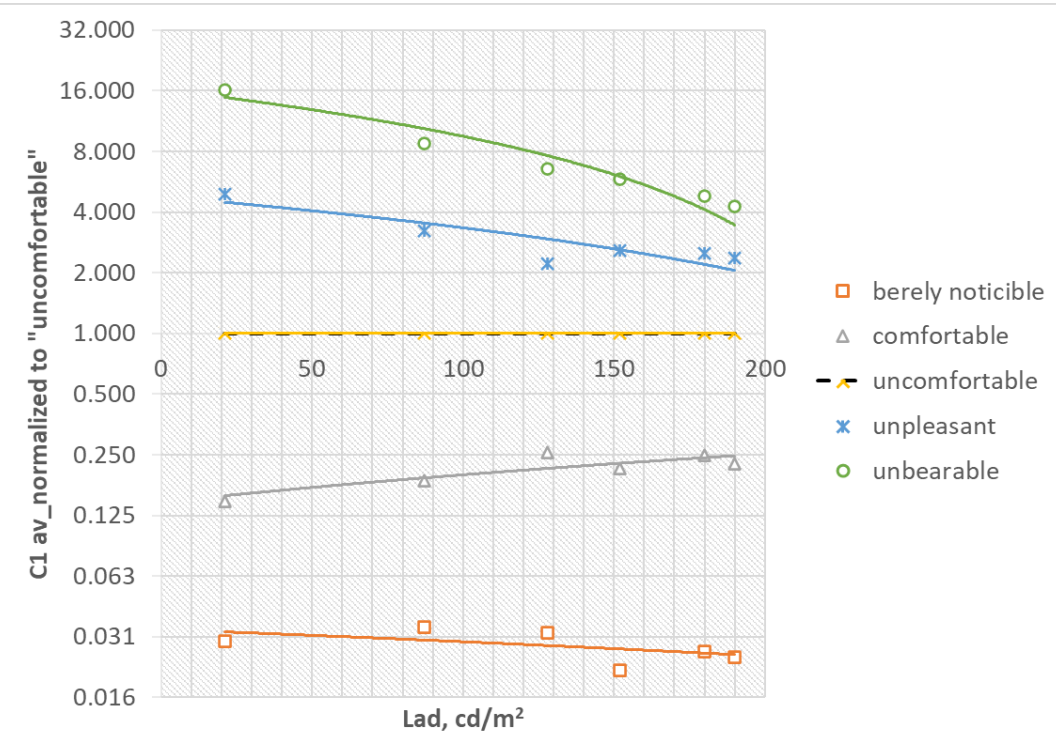

Figure 4: The distance between the average contrasts $C_{1}$ for different sensations, depending on the adaptation luminance

The obtained threshold values of contrasts, see Table 2, found in the space of the visual perception scale can be further used as threshold values for evaluating the continuous SADL of lighting across the scene in a new quality criterion for simple visual tasks, such as reading a sign or viewing the surrounding space.

Table 2

Threshold contrasts of sensations relative to the axis of discomfort for different levels of luminance of adaptation

\begin{tabular}{cccccc}
\hline $\begin{array}{c}\text { Lad, } \\
\mathrm{cd} / \mathrm{m} 2\end{array}$ & $\begin{array}{c}\text { barely } \\
\text { noticeable }\end{array}$ & comfortable & uncomfortable & unpleasant & unbearable \\
\hline 21 & 0.030 & 0.147 & 1.000 & 4.906 & 16.245 \\
87.0 & 0.035 & 0.185 & 1.000 & 3.246 & 8.746 \\
128.0 & 0.033 & 0.257 & 1.000 & 2.224 & 6.528 \\
152.0 & 0.021 & 0.214 & 1.000 & 2.567 & 5.809 \\
180.0 & 0.026 & 0.249 & 1.000 & 2.490 & 4.828 \\
190.0 & 0.025 & 0.225 & 1.000 & 2.376 & 4.278 \\
\hline
\end{tabular}

\section{Conclusion}

As a result of the work conducted, a new scale of visual perception was built by luminance contrasts with a probability of $50 \%$. That is, for $50 \%$ of observers, with a certain contrast, one or another sensation will arise according to the categories of the constructed scale. This scale can be used to find the quality of indoor lighting by SADL, expanding the functionality of modern methods of modelling CG. This scale reflects the relationship between sensations compared to the contrast that causes a sense of discomfort. This sensation is the simplest for observers and is in the centre of the scale. Therefore, "uncomfortable" or uncomfortable contrast is chosen as the centre of reference. This scale is obtained for a simple classical case-the observation of a glare source on a uniform background. Nevertheless, it allows us to find the nature of the relationship between sensations in numbers. Therefore, these values can be used to evaluate the lighting quality of simple scenes with a similar luminance distribution, that is, with a single source in the field of view. Thus, the first approximation to the assessment of the quality of lighting according to the SADL is made. 
Typical office visual tasks while working at a computer are different from reading a sign or seeing the surrounding space. Therefore, the next stage of the study will be an experiment to assess the lighting conditions during the performance of typical office visual tasks.

Three experiments are planned:

- $\quad$ reading black text on a white sheet installed on a turned-off computer screen;

- $\quad$ reading black text on the white screen of the included computer screen;

- reading white text on the black screen of the turned-on computer screen.

All three visual tasks are selected as typical office tasks, that is, imitation of reading printed documentation on white sheets of paper, the work of an editor and the work of a designer in the AutoCAD program. Conducting this experiment will expand the functionality of the new scale of visual perception and will allow evaluating a larger number of lighting scenes according to the SADL at the modelling stage in the CG programs.

\section{Acknowledgements}

The authors would like to express their gratitude to the students of the Department of Lighting Engineering E. Vasiliev, I. Ryazantsev, D. Budchenkov, R. Makarenko, V. Zarzhetsky, A. Shubina, D. Tverdov, S. Kushagra and S. Chuprasov for their participation in the experiment.

\section{References}

[1] D. Neumann (Ed.), The Structure of Light: Richard Kelly and the Illumination of Modern Architecture, New Haven, CT: Yale University Press, 2011.

[2] P. R Boyce, Human Factors in Lighting 3rd Edition, CRC Press, 2014. doi: 10.1201/b16707.

[3] K. Broszio, M. Knoop, M. Niedling, S. Volker, Spatial distribution of lighting scenes and its impact on non-visual effects. 2017, Conference: The Society for Light Treatment and Biological Rhythms (SLTBR) annual meeting.

[4] V.P. Budak, V.S. Zheltov, T.V. Meshkova, R.Sh. Notfullin, Evaluation of illumination quality based on spatial-angular luminance distribution, Light \& Engineering 25(4) (2017) 24-31.

[5] J.E. Saunders, The role of the level and diversity of horizontal illumination in an appraisal of a simple office task, Lighting Res. Technol. 1 (1969) 37-46.

[6] A.I. Slater, M.J. Perry, D.J. Carter, Illuminance differences between desks: Limits of acceptability, Lighting Res. Technol. 25 (1993) 91-103.

[7] G.H. Scheir, P. Hanselaer, P. Bracke, G. Deconinck, W.R. Ryckaert, Calculation of the Unified Glare Rating based on luminance maps for uniform and non-uniform light sources, Building and Environment 84 (2015) 60-67.

[8] M. Luckiesh, S.K. Guth, Luminance in the visual field at borderline between comfort and discomfort, Illuminating Engineering 44(11) (1949) 650-670.

[9] M. Luckiesh, L.L. Holladay, Glare and Visibility, Transactions of the IES 20 (1925) 221-252.

[10] W. Bommel, Interior Lighting. Fundamentals, Technology and Application, Springer, Cham, 2019. doi:10.1007/978-3-030-17195-7.

[11] V. P. Budak, E. I. Ilyina, Construction of a psychophysical scale of visual comfort of lighting based on a neural network: preparation of an experiment, Lighting Engineering 2 (2021) 30-36.

[12] G. G. Kotik, A. B. Matveev, V. V. Pereima, I. L. Tokhadze, Categorical quality assessments and their interrelation on the psychophysical scale, Lighting Engineering 3 (1975) 3-5.

[13] V. P. Budak, V. S. Zheltov, T. V. Meshkova, V. D. Chembaev, A new criterion for lighting quality and its approbation in laboratory conditions, Bulletin of the MEI 1 (2020) 73-81. 